\title{
Fullerenes Enhance Self-Assembly and Electron Injection of Photosystem I in Biophotovoltaic Devices
}

\author{
Nahid Torabi, Xinkai Qiu, Manuel López-Ortiz, Mark Loznik, Andreas Herrmann, Ahmad Kermanpur, \\ Ali Ashrafi, and Ryan C. Chiechi*
}

Cite This: Langmuir 2021, 37, 11465-11473

Read Online

ABSTRACT: This paper describes the fabrication of microfluidic devices with a focus on controlling the orientation of photosystem I (PSI) complexes, which directly affects the performance of biophotovoltaic devices by maximizing the efficiency of the extraction of electron/hole pairs from the complexes. The surface chemistry of the electrode on which the complexes assemble plays a critical role in their orientation. We compared the degree of orientation on self-assembled monolayers of phenyl- $\mathrm{C}_{61}$-butyric acid and a custom peptide on nanostructured gold electrodes. Biophotovoltaic devices fabricated with the $\mathrm{C}_{61}$ fulleroid exhibit significantly improved performance and reproducibility compared to those utilizing the peptide, yielding a 1.6-fold increase in efficiency. In addition, the $\mathrm{C}_{61}$-based devices were more stable

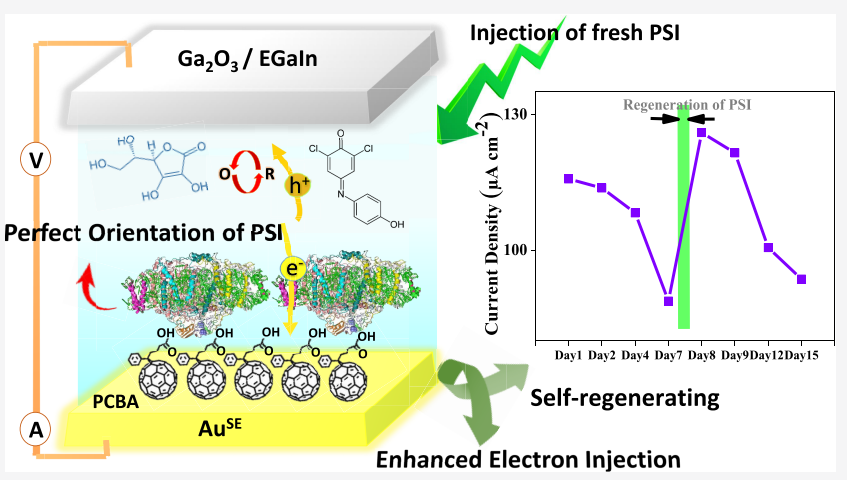
under continuous illumination. Our findings show that fulleroids, which are well-known acceptor materials in organic photovoltaic devices, facilitate the extraction of electrons from PSI complexes without sacrificing control over the orientation of the complexes, highlighting this combination of traditional organic semiconductors with biomolecules as a viable approach to coopting natural photosynthetic systems for use in solar cells.

\section{INTRODUCTION}

In artificial solar cells, the electron/hole pairs generated by the absorption of photons must be separated before they recombine, which is typically accomplished architecturally with heterojunctions or $\mathrm{p}-\mathrm{n}$ junctions. ${ }^{1}$ Control over these structures is vital to making efficient solar cells and is a central challenge in organic/hybrid photovoltaics, which are desirable for their mechanical compliance and processability from the solution. ${ }^{2,3}$ Biophotovoltaic (BPV) devices are solar cells that utilize the components of natural photosynthetic proteins such as photosystem I/II (PSI/II) from, for example, algae, plants, or cyanobacteria to effect photon-induced charge separation. When light is absorbed by PSI in a photosynthetic organism, a series of reactions take place that converts photon energy into chemical reducing potential. The initial step, however, is identical to that of artificial solar cells; PSI converts photon energy into spatially separated electron/hole pairs. However, it does so with $\sim 100 \%$ internal quantum efficiency by effectively building a precise, nanoscale junction into each protein complex. Chromophores surrounding a reaction center at the center of each PSI protein pass an exciton into an electron transport chain that moves charges down the redox potential of the reaction center, creating a driving force for the transfer of an electron from the $P_{700}$ unit on one side to the $F_{A} / F_{B}$ sites on the other, making PSI behave as a photodiode. ${ }^{4}$ The natural abundance and ease of isolation of these complexes, particularly PSI, make them ideal candidates for integration in biophotovoltaic devices. ${ }^{4-8}$ These remarkable nanophotoelectric machines have been used in solid-state BPV devices $^{9-13}$ and dye-sensitized photoelectrochemical cells. $^{14-17}$ In living organisms, PSI/II complexes are continually refreshed as they degrade, which is not possible in solid-state devices. This fundamental limitation is the reason that we are particularly interested in dye-sensitized BPV devices, where PSI can be refreshed simply by circulating through the device. ${ }^{15}$

Several parameters affect the power generation of dyesensitized BPVs, such as choosing appropriate working electrodes, an electrolyte to maintain a stable environment for ion exchange, and a redox mediator to shuttle charges away from the PSI complexes. ${ }^{18}$ There are different approaches to improving and maximizing the performance of BPV devices, such as the modification of electrode morphologies to increase

Received: June 8, 2021

Revised: September 6, 2021

Published: September 21, 2021 
the surface area over which light can be absorbed, ${ }^{16}$ wiring photosynthetic complexes with nanoscale materials, ${ }^{19,20}$ and the integration of carbon nanotubes. ${ }^{21,22}$ The efficiency of BPV cells is still low compared to artificial solar cells, thus necessitating a deeper understanding of the contributions of each of these parameters.

This paper focuses on the direct extraction of charges by the electrode that is in physical contact with the PSI complexes. The orientation of the PSI complexes, among others, is the most important parameter because it aligns the $\mathrm{P}_{700}$ and $\mathrm{F}_{\mathrm{A}} / \mathrm{F}_{\mathrm{B}}$ sites to manifest efficient injection of electrons and holes into the external circuit through the correct pathways. ${ }^{13}$ However, PSI is a membrane protein that does not evolve to facilitate a preferred orientation on metallic electrodes. It rather tends to assemble into isotropic, heterogeneous monolayers and multilayers, which is detrimental to BPV performance. ${ }^{10}$ Recent studies have investigated anisotropic monolayers that use various linker strategies to modify the surface of the electrode, ${ }^{13,23-26}$ coupling to plasmonically active metallic nanowires, ${ }^{27}$ short peptides developed by phage display, ${ }^{28}$ or conductive interlayers ${ }^{15,29-32}$ to bias the orientation of PSI. Even perfectly oriented PSI, however, must also be free of traps and electronically coupled to the electrode or else the electron/hole pairs will recombine before they can be extracted. $^{33-37}$ Thus, an ideal strategy for controlling the orientation of PSI must also modify the electrode-protein interface for optimal alignment between the energy levels of $\mathrm{P}_{700}$ or $\mathrm{F}_{\mathrm{A}} / \mathrm{F}_{\mathrm{B}}$ sites and the work function of the electrode.

We used phenyl- $\mathrm{C}_{61}$-butyric acid (PCBA) ${ }^{38}$ and a peptide (IQAc, sequence: IQAGKTEHLAPDC in which a cysteine residue was attached to the C-terminus) ${ }^{39}$ as linkers to orient PSI trimers on $\mathrm{Au}$ electrodes and compared their influence on the efficiency and lifetime of dye-sensitized BPVs. Substituted fulleroids form densely packed self-assembled monolayers (SAMs) on Au with their terminal groups, carboxylic acid in the case of PCBA, oriented toward the ambient interface. ${ }^{40}$ The peptide sequence is designed to bind selectively to one side of PSI using phage display ${ }^{28}$ and includes a terminal cystine to facilitate the formation of SAMs on Au. Electrodes functionalized with carboxylic acid groups bias the selfassembly of PSI to prefer orienting the $\mathrm{F}_{\mathrm{A}} / \mathrm{F}_{\mathrm{B}}$ sites toward the electrode. ${ }^{41-44}$ Thus, both linkers favor the injection of electrons into the electrode, but PCBA mitigates the injection barrier with a fullerene cage.

\section{EXPERIMENTAL SECTION}

Photosystem I Preparation and Purification. Photosystem I started by growing the thermophilic cyanobacterium Thermosynechococcus elongatus BP-1 in BG11 medium. ${ }^{45}$ Using a protocol adapted from El-Mohsnawy and Mukherjee et al., thylakoid membranes were prepared. $^{8,46}$ Subsequently, additional purification steps were performed using chromatographic purification. Complete information about the whole process, chlorophyll a determination, and protein concentration estimation are available in previously published work. ${ }^{15}$

Fabrication of Soft Devices. Designing of microfluidic channels to fabricate the unique experimental platform was done using soft lithography technology. All steps of fabrication of microfluidic channels were previously described. ${ }^{15}$ Briefly, after pattern design and mask fabrication, a master mold for poly(dimethylsiloxane) (PDMS) using a negative photoresist of SU-8 was fabricated on a $\mathrm{Si}$ wafer by UV lithography. The resulting microchannel PDMS chip was then reversibly attached to the bottom PDMS to create a microfluidic device. For increasing the surface area for immobilization of PSI, we chose a Whatman Anodisc AAO membrane to produce arrays of nanotubes and evaporated $150 \mathrm{~nm}$ of Au against the rotating platform at a $45^{\circ}$ incident angle by electron beam evaporation to control the heights of the nanotubes. After it was finished, we applied a strip of $\mathrm{Au}^{\mathrm{SE}}$ onto the bottom PDMS. The total surface area of $\mathrm{Au}^{\mathrm{SE}}$ that was exposed under the illumination was estimated as previously described. ${ }^{15}$

IQAc Peptide Design. The peptide IQAc (sequence: IQAGKTEHLAPDC) was designed as described in detail in ref 39. The peptide was synthesized according to standard procedures employing fmoc chemistry. Briefly, by adapting IQA peptides generated by Gordiichuk et al., ${ }^{28}$ we developed these peptides to functionalize $T$. elongatus PSI stromal side to electrodes, and the C-terminal phosphorylated serine residue in IQA was replaced by a cysteine in IQAc.

Fabrication of BPV Devices Based on the Bilayer of PCBA/ PSI. After evaporating $150 \mathrm{~nm}$ of Au onto the AAO Whatman Anodisc membranes at an angle of $45^{\circ}$ using an electron beam evaporator, fabrication of PCBA-based biophotovoltaic devices started. A stripe of $15 \mathrm{~mm} \times 1 \mathrm{~mm}$ of $\mathrm{Au}^{\mathrm{SE}}$ was placed onto the bottom PDMS and etched by drop-casting $1 \mathrm{M} \mathrm{NaOH}$ for $1 \mathrm{~h}$. After rinsing with deionized water and ethanol and drying with a gentle nitrogen flow, SAMs of PCBA were produced onto $\mathrm{Au}^{\mathrm{SE}}$ by incubation of $\mathrm{Au}^{\mathrm{SE}}$ in a $\mathrm{N}$-methyl-2-pyrrolidone (NMP) solution of PCBA for $12 \mathrm{~h}$ and subsequently the $\mathrm{Au}^{\mathrm{SE}}$ electrode was rinsed with NMP three times and dried with a nitrogen flow. Then, we immersed it in an aqueous solution of PSI trimers $(0.8 \mu \mathrm{M})$ for $6 \mathrm{~h}$ to immobilize PSI onto the electrode. After $6 \mathrm{~h}$, the $\mathrm{Au}^{\mathrm{SE}}$ electrode was rinsed with deionized water three times and dried with a nitrogen flow gently. Then, using an optical microscope, we adjusted one of the channels of the top microfluidic channels somehow surrounded by the $\mathrm{Au}$ electrode. Finally, we completed our devices using redox couples as an electrolyte that is loaded to the channel in contact with $\mathrm{Au}^{\mathrm{SE}}$, and eutectic Ga-In (EGaIn) is loaded into the channel without contact with $\mathrm{Au}^{\mathrm{SE}}$.

Fabrication of BPV Devices Based on the Monolayer of IQAc-PSI. Fabrication of IQAc-based biophotovoltaic devices followed the same procedures as PCBA-based biophotovoltaic devices. The difference here is that instead of a bilayer of PCBA/ PSI, we prepared a mixture solution of IQAc peptides and PSI with a ratio of 10:1 to ensure that all PSI complexes are bound with IQAc peptides. Then, we immersed $\mathrm{Au}^{\mathrm{SE}}$ in that mixture solution for $8 \mathrm{~h}$. Then, $\mathrm{Au}^{\mathrm{SE}}$ was rinsed with deionized water three times and dried under a gentle flow of nitrogen, and the device was completed like PCBA-based BPV devices.

Photocurrent Measurements. Electrical measurements were performed inside a Faraday cage with a measuring setup for both bottom gold and EGaIn electrodes along with a Keithley source meter. For all measurements to prevent overheating, first, we let the device cool down to $0{ }^{\circ} \mathrm{C}$, and after the temperature stabilized, measurements were taken. Each device was illuminated using a 655 $\mathrm{nm}$ laser source, which was installed $9 \mathrm{~cm}$ above the device and emitted light intensity of $62 \mathrm{~mW}$ with an effective area that was previously explained. ${ }^{15}$ Photochronoamperometric measurements were performed with a small bias under light and dark cycles within the different on and off periods of the laser source. $J-V$ curves were produced in a bias window from -0.2 to $0.8 \mathrm{~V}(0 \rightarrow 0.8 \rightarrow 0 \rightarrow-0.2$ $\rightarrow 0 \mathrm{~V}$ ) with a step size of $0.01 \mathrm{~V}$. For all measurements, a scan size of $0.01 \mathrm{~V}$ was used.

\section{RESULTS AND DISCUSSION}

Device Design. The basic design of the BPV devices used to investigate the PCBA and IQAc linkers is a co-fabricated microfluidic channe ${ }^{47}$ that defines an electrode comprising the liquid metal eutectic Ga-In (EGaIn), which is in contact with the electrolytic medium. The floor of the microfluidic channel, which is perpendicular to the EGaIn electrode, supports a nanostructured gold electrode $\left(\mathrm{Au}^{\mathrm{SE}}\right)$ fabricated by shadow evaporation. ${ }^{48}$ The design of this prototype device is a simplified version of the one we published previously, ${ }^{15}$ as shown in Figure 1. The device architecture exploits the self- 

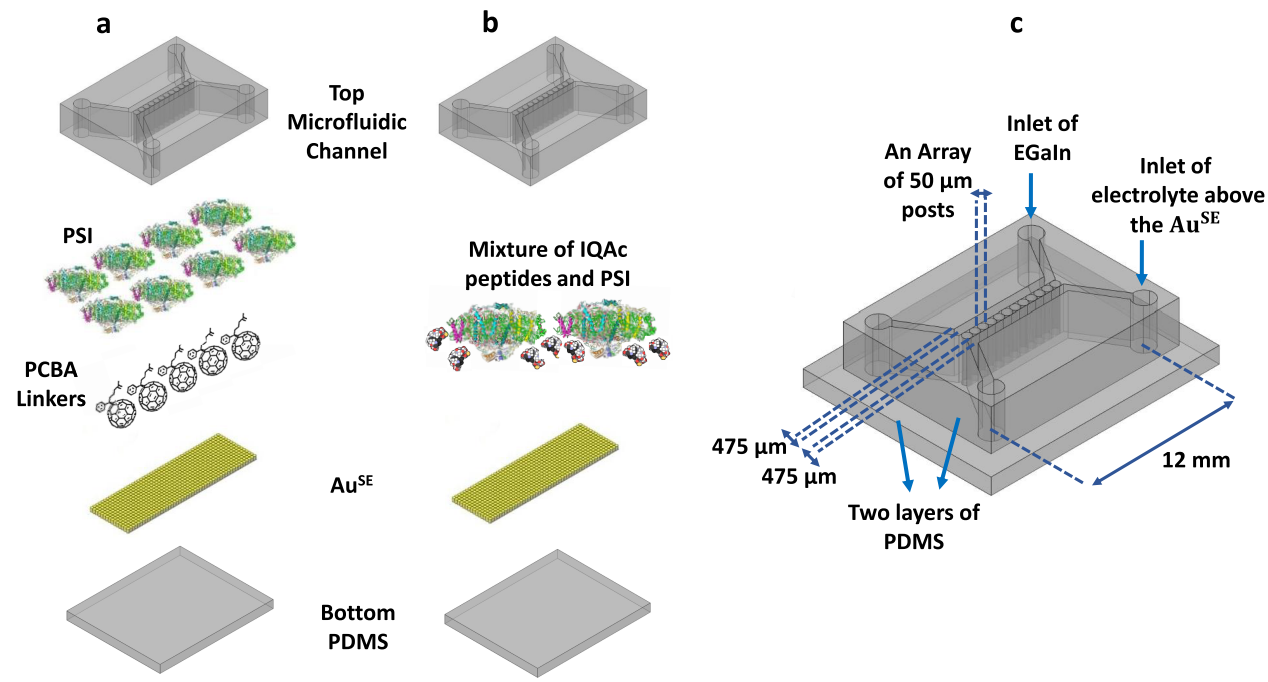

Figure 1. Schematic of the two configurations of the biophotovoltaic test devices, (a) incorporating a bilayer of PCBA/PSI and (b) incorporating a monolayer of IQAc-PSI. In both configurations, the $\mathrm{Au}^{\mathrm{SE}}$ electrode is covered by the channel into which EGaIn and electrolyte are sequentially injected. (c) Schematic of an assembled test device showing a $12 \mathrm{~mm}$ central channel, which is connected to inlets for injecting EGaIn and electrolyte into either side of an array of $50 \mu \mathrm{m}$ posts spaced by $50 \mu \mathrm{m}$. The total width of the central channel is $475 \mu \mathrm{m}$.

a

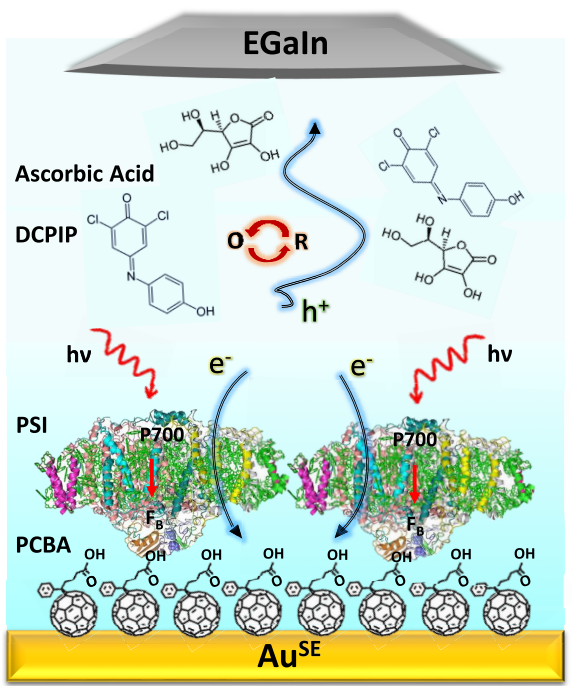

b

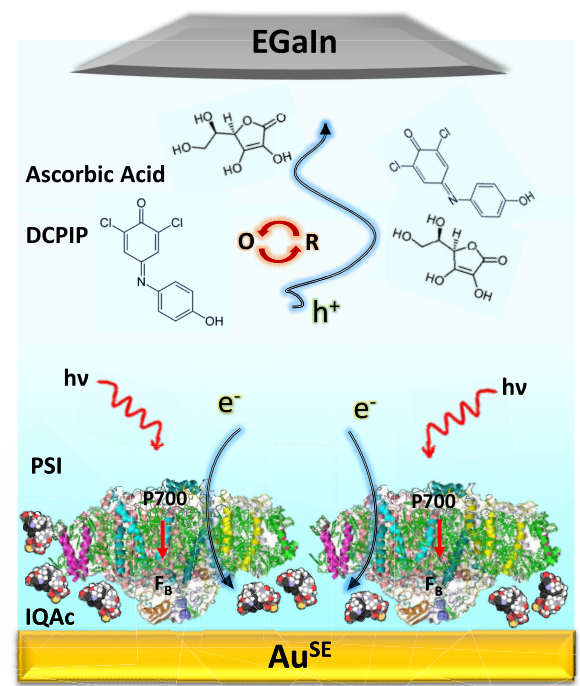

Figure 2. Working principle of (a) PCBA-based and (b) IQAc-based biophotovoltaic devices. For PCBA-based devices, a SAM of PCBA is first grown on the $\mathrm{Au}^{\mathrm{SE}}$ electrode, which directs the subsequent self-assembly of PSI with the stromal side toward the PCBA. For the IQAc peptidebased devices, PSI is first incubated with IQAc, which preferentially binds to the stromal side. A cystine residue then directs the assembly of the IQAc-PSI complexes on the surface of the $\mathrm{Au}^{\mathrm{SE}}$ electrode. For both configurations, the devices are completed by the injection of EGaIn electrodes and a solution of DCPIP and ascorbic acid. Under illumination, this redox couple shuttles holes from the PSI and the EGaIn electrode, while electrons are injected into PCBA or, in the case of the IQAc-based devices, directly into $\mathrm{Au}^{\mathrm{SE}}$.

assembly of PSI complexes on Au both for ease of fabrication and to allow the PSI to be replenished in operando. To maximize the photocurrent, we use $\mathrm{Au}^{\mathrm{SE}}$, which is fabricated by evaporating $\mathrm{Au}$ onto rotating, porous membranes (Whatman Anodisc) at an angle of $45^{\circ}$, resulting in high-surface-area $\mathrm{Au}^{\mathrm{SE}}$ electrodes after etching the membrane of $\mathrm{Au}^{\mathrm{SE}}$ by drop-casting $1 \mathrm{M} \mathrm{NaOH}$ for $1 \mathrm{~h}$. The surface of the $\mathrm{Au}^{\mathrm{SE}}$ electrodes contains thin-walled nanotubes of $\mathrm{Au}$ that are wide enough for the inner and outer walls to be covered completely by PSI. The self-assembly of PSI is guided either by IQAc or PCBA as follows. For IQAc, complexes are first formed in solution from a mixture of IQAc peptides and PSI with a ratio of 10:1 to ensure that all PSI complexes are bound with IQAc peptides.
Dried $\mathrm{Au}^{\mathrm{SE}}$ substrates are then immersed in the IQAc-PSI solution for $8 \mathrm{~h}$ to ensure the formation of a densely packed mixed monolayer of IQAc-PSI. The IQAc peptides adhere selectively to a small area on the $\mathrm{F}_{\mathrm{A}} / \mathrm{F}_{\mathrm{B}}$ side of the PSI complexes (by recognizing the $\mathrm{Fe}_{4} \mathrm{~S}_{4}$ cluster), anchoring them to the surface of the $\mathrm{Au}^{\mathrm{SE}}$ electrodes through the cystine anchor; the $\mathrm{Fe}_{4} \mathrm{~S}_{4}$ terminus of PSI is in direct contact with the electrode, and thus IQAc-PSI tests the efficiency of direct electron injection in the near-perfect (98\%) orientation of SAMs of PSI. To fabricate devices with PCBA linkers, SAMs of PCBA are first grown in $\mathrm{Au}^{\mathrm{SE}}$ and then rinsed with NMP and allowed to dry. The PCBA-modified $\mathrm{Au}^{\mathrm{SE}}$ electrodes are then immersed in a solution of PSI, which forms SAMs on top of 


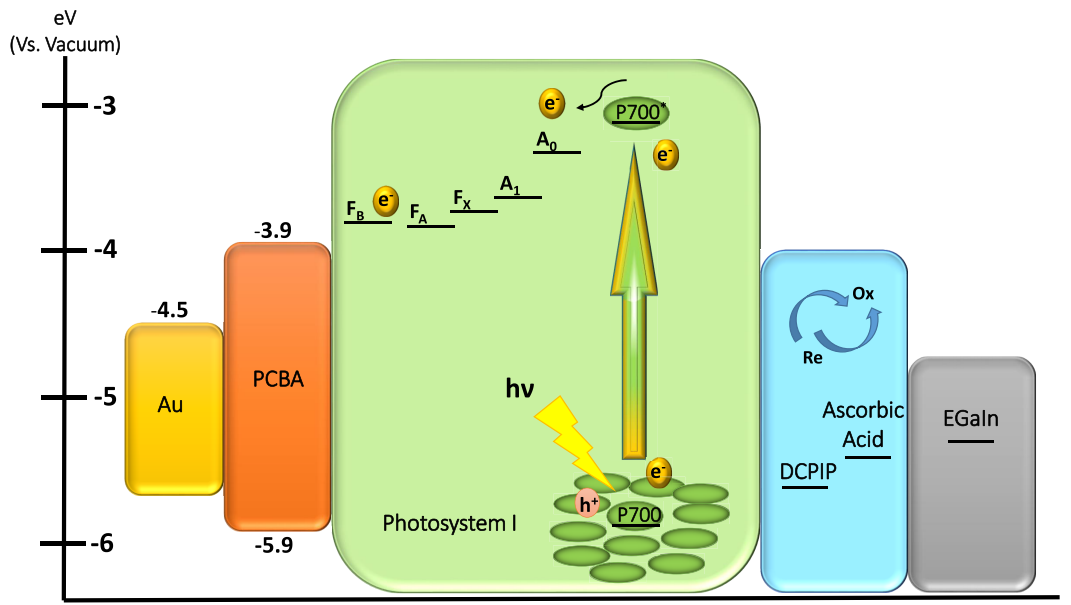

Figure 3. Energy level and schematic representation of the final PCBA-based BPV device. (The energy level data are given elsewhere. ${ }^{53-55}$ ).

the PCBA assembly that are directed by the terminal carboxylate groups; the terminus of PSI entirely contacts PCBA-Au ${ }^{\mathrm{SE}}$, and thus PSI-PCBA tests the efficiency of electron injection mediated by fullerenes in the near-perfect orientation of SAMs of PSI (Figures S1 and S2).

In both configurations, the devices are completed by covering the modified $\mathrm{Au}^{\mathrm{SE}}$ with a specially designed poly(dimethylsiloxane) (PDMS) microfluidic channel, in which the counter electrode is defined by EGaIn confined in the channel next to the $\mathrm{Au}^{\mathrm{SE}}$ electrode and the electrolyte comprising the redox couple comprising the remaining volume. This process is explained in detail in ref 15 . For this study, we chose a combination of 2,6-dichlorophenolindophenol (DCPIP, $0.1 \mathrm{mM}$ ) and ascorbic acid $(5 \mathrm{mM})$ as redox mediators. ${ }^{32}$ Both components are essential for transporting holes from the oriented PSI to the EGaIn electrode, and each plays an important role in the overall performance of the device. While there is certainly room for improvement, this paper focuses on the extraction of electrons from PSI and makes no attempt to further optimize the redox couple. Figure 2 schematically shows the charge flow and orientation of the PSI complex in the completed devices. When light strikes the SAMs of PSI, the resulting excitons are collected by the $\mathrm{P}_{700}$ reaction center, which is immediately oxidized to $\mathrm{P}_{700}^{+}$by $\mathrm{A}_{0}$, which then relaxes the electron along the $A_{1} / F_{X} / F_{A} / F_{B}$ chain, where it is localized on the exposed $\mathrm{Fe}_{4} \mathrm{~S}_{4}$ cluster. This electron is either injected directly into $\mathrm{Au}^{\mathrm{SE}}$ (IQAc-PSI) or a fullerene (PCBA/PSI), while the hole that is localized on $\mathrm{P}_{700}^{+}$, which sits on the ambient side of the SAM, is transferred to DCPIP and shuttled to the EGaIn electrode, assisted by ascorbic acid. ${ }^{16,49}$ Fullerenes are well known as efficient acceptors/ntype semiconductors; ${ }^{50-52}$ thus, PCBA should readily accept electrons from $\mathrm{Fe}_{4} \mathrm{~S}_{4}\left(\mathrm{~F}_{\mathrm{B}}\right)$ as shown in Figure 3, effectively playing the role that ferredoxin plays on the stromal side of the thylakoid membrane.

One of the advantages of fullerenes is that they can be readily functionalized without substantially affecting their electronic properties; thus, PCBA is functionally nearly identical to the more well-known methanofullerene acceptor $\mathrm{PCBM}^{56-59}$ but with a polar, protic carboxylic acid group at the end of the pendant chain. The chemisorption of fullerenes on $\mathrm{Au}^{60}$ to form densely packed SAMs is similarly unaffected, resulting in SAMs with fullerene anchors and carboxylic acid groups at the ambient interface. ${ }^{40}$ Modification of the surface of $\mathrm{Au}^{\mathrm{SE}}$, therefore, modifies the work function (via the fullerenes) and presents a polar, protic surface (via the carboxylic acid groups) onto which PSI can assemble, favoring the orientation with the $\mathrm{Fe}_{4} \mathrm{~S}_{4}$ cluster pointing into the surface. ${ }^{41-44}$ In our previous work, we used thiol-SAMs to modify $\mathrm{Au}$ substrates with polar and protic groups and unambiguously established the resulting absolute orientations of PSI complexes assembled on top of them. ${ }^{29}$ However, this approach places a tunneling barrier between the PSI and the electrode. ${ }^{15}$ Here, we avoid that barrier by either directly contacting $\mathrm{Au}^{\mathrm{SE}}$ with PSI (IQAc-PSI) or coupling PSI to $\mathrm{Au}^{\mathrm{SE}}$ (PCBA/PSI). Fullerene-SAMs are also much more (electro)chemically stable than thiol-SAMs, making them more suitable for electrode modification in BPV devices. In our previous work, we used phage display to design a peptide sequence (IQA) capable of selectively binding the $\mathrm{F}_{\mathrm{A}} / \mathrm{F}_{\mathrm{B}}$ side of PSI, leading to the near-perfect orientation in SAMs on transparent metal oxide surfaces. ${ }^{28}$ We adapted these peptides to $\mathrm{Au}$ surfaces by installing a cysteine; IQAc has the same sequence as IQA but with a C-terminal cystine residue capable of forming $\mathrm{S}-\mathrm{Au}$ bonds.

Device Performance. Photocurrent responses of BPV devices incorporating SAMs of PCBA/PSI and IQAc-PSI in the dark and under illumination are shown in Figures 4 and 5, respectively. Extracted parameters of the BPV devices comprising PCBA/PSI and IQAc-PSI such as open-circuit voltage $\left(V_{\mathrm{OC}}\right)$, short-circuit current density $\left(J_{\mathrm{SC}}\right)$, fill factor (FF), maximum output power $\left(P_{\max }\right)$, and external power conversion efficiency $(\eta)$ are summarized in Table 1 . The devices comprising PCBA/PSI performed significantly better in every metric, which we ascribe to decreased recombination losses, the effective dipole-supported charge separation, and increased electron and hole densities at the interfaces; the larger difference between the quasi-Fermi levels of electrons and holes, in particular, likely increases $V_{\mathrm{OC}}$ and FF. The photocurrent from BPV devices incorporating SAMs of PCBA/PSI was also more stable under constant illumination than those comprising IQAc-PSI; although the average output was stable over $120 \mathrm{~min}$, it fluctuated considerably more for IQAc-PSI than PCBA/PSI (Figure S7). Figure S8 shows absorbance spectra of PCBA/PSI devices, which were measured before $(0 \mathrm{~min})$ and after $(120 \mathrm{~min})$ under light illumination, thereby characteristic peaks of PSI remaining stable after long exposure to light irradiation. Control devices 

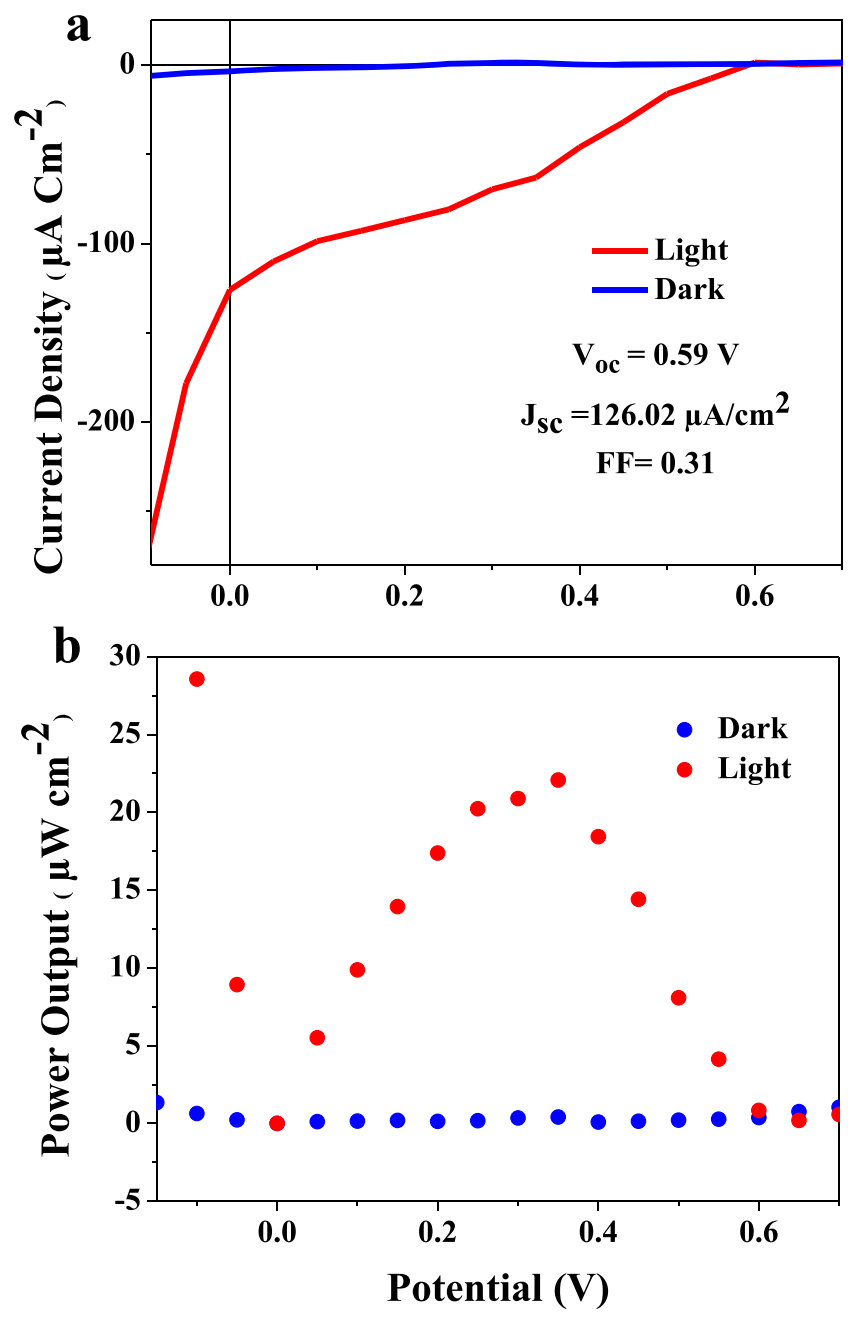

Figure 4. (a) Current density and (b) output power of a typical BPV device fabricated using a bilayer of PCBA/PSI in the dark and under illumination

either lacking PSI or using boiled (deactivated) PSI (Figure S3 and Table S1) generated negligible photocurrent. ${ }^{61,62}$

Figure 6 compares photochronoamperometric measurements for devices comprising PCBA/PSI (black) and IQAcPSI (red). The comparison of $J_{S C}$ was done under repeated cycles of dark $(4 \mathrm{~min})$ and illumination $(1 \mathrm{~min})$, indicated with light pink bars. Please note that the photochronoamperometric measurements were performed with a small bias and on the best-performing devices as shown in Figures 6, S7, and S9. The devices comprising PCBA/PSI and IQAc-PSI yielded stable values of $J_{\mathrm{SC}}\left(\mu \mathrm{A} / \mathrm{cm}^{2}\right)$ of 394 and 257 , respectively. Figure 6 also compares control devices with only PCBA (green) and only IQAc (blue), yielding stable values of $J_{\mathrm{SC}}\left(\mu \mathrm{A} / \mathrm{cm}^{2}\right)$ of 132 and 85 , respectively. These measurements confirm that PSI generates substantial photocurrent, which is larger than that from direct irradiation of the electrodes/linkers. The photocurrent density of BVP devices comprising PCBA/PSI remained stable after two cycles of illumination and then decreased around $14 \%$ but remained stable for the remaining illumination cycles. The BPV devices comprising IQAc-PSI, however, decayed gradually after each light/dark cycle, amounting to an overall decrease of $15 \%$. Photochronoamperometric measurements over $150 \mathrm{~min}$ (Figure S9), however, reveal a constant degradation for both devices, suggesting that
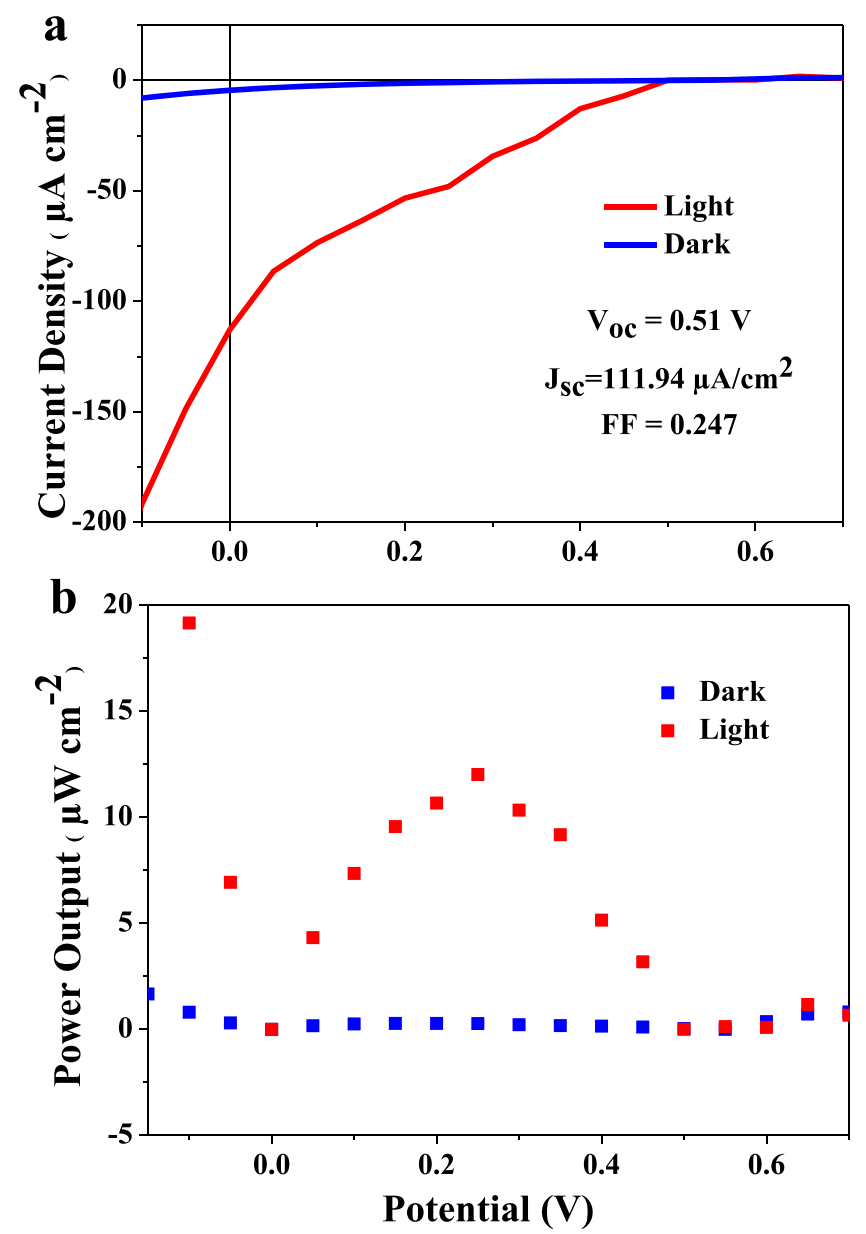

Figure 5. (a) Current density and (a) output power of a typical BPV device fabricated using a monolayer of IQAc-PSI in the dark and under illumination.

Table 1. Comparison of Various Parameters of BPV Devices Fabricated Using a Bilayer of PCBA/PSI and a Monolayer of IQAc-PSI

$\begin{array}{lccccc} & V_{\mathrm{OC}}(\mathrm{V}) & \begin{array}{c}J_{\mathrm{SC}} \\ \left(\mu \mathrm{A} / \mathrm{cm}^{2}\right)\end{array} & \mathrm{FF} & \begin{array}{c}P_{\max } \\ \left(\mu \mathrm{W} / \mathrm{cm}^{2}\right)\end{array} & \eta(\%) \\ \text { PCBA/PSI } & 0.59 & 126.02 & 0.31 & 22.07 & 0.0043 \\ \text { IQAc-PSI } & 0.51 & 111.94 & 0.25 & 12.02 & 0.0027\end{array}$

the short-term trends in Figure 6 are related to the linkers (PCBA and IQAc), while the long-term degradation is related to the deactivation of PSI. This deactivation of PSI under illumination is one of the challenges of working with natural photosystems, which undergo photoinhibition ${ }^{63}$ on a similar timescale due to photodamage. ${ }^{64}$ All of our measurements were performed under a $655 \mathrm{~nm}$ laser source, which gives the highest photocurrent response of our BPV devices. The electrical response of our devices under solar illumination was below the noise level due to the physical size of the devices and low incident light absorption of the monolayer of PSI complexes, whereas the findings from other studies represent that the absorption spectra of photosynthetic protein complexes span between the band attributable to the characteristic absorption peaks of pigment proteins (Figure S8), which is in good agreement with the external quantum efficiency (EQE) of other biophotovoltaic devices. ${ }^{65-67}$ 


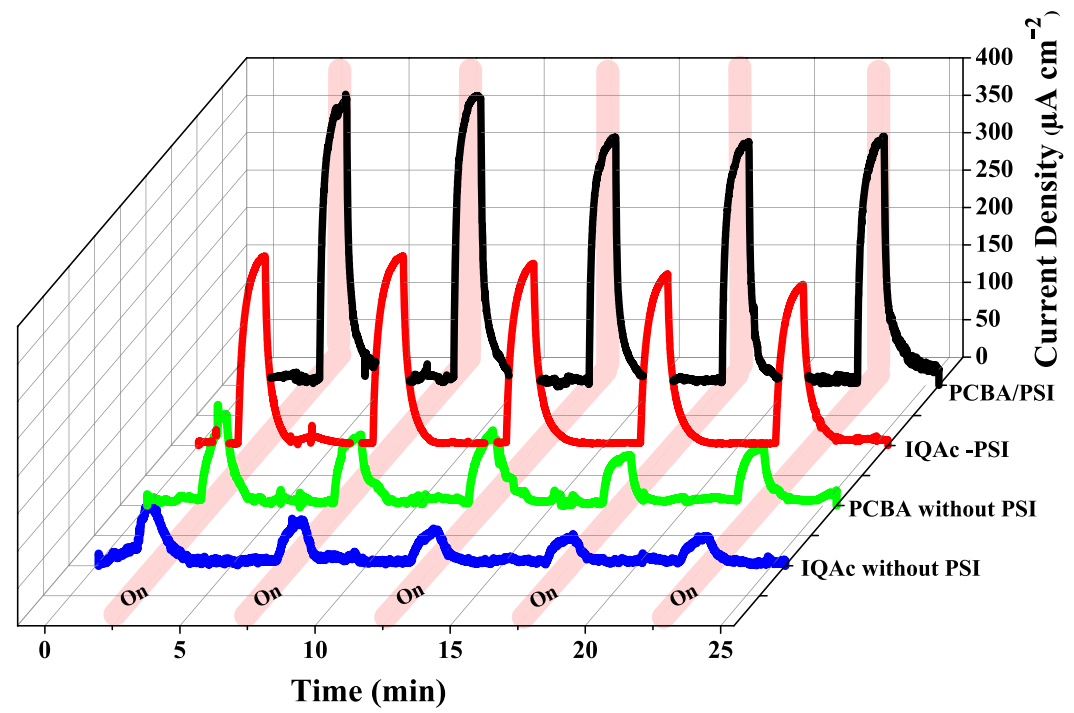

Figure 6. Comparison of $J_{\mathrm{SC}}$ between four best-performing devices fabricated by a bilayer of PCBA/PSI (black), a monolayer of the mixture of IQAc peptide and PSI (red), a monolayer of PCBA without PSI (green), and a monolayer of IQAc peptide without PSI (blue) under cycles of illumination $(1 \mathrm{~min})$ and dark $(4 \mathrm{~min})$ over a total of $25 \mathrm{~min}$. The periods in which the devices are illuminated are specified by light pink bars.

As discussed above, a key feature of our BVP device design is that PSI can be replenished in operando, mimicking the normal turnover of membrane-bound complexes that occurs in organisms. ${ }^{64}$ This replenishment exploits the ability of SAMs to undergo in-place exchange, i.e., when a SAM is exposed to a solution containing free molecules, the system returns to the equilibrium state of a pure SAM comprising the free molecules. Thus, the BVP devices are self-regenerating as long as fresh PSI is circulated through their microfluidic channel(s). To determine how IQAc and PCBA affect the regeneration process and to evaluate the stability of the devices over the course of several days, we measured $J-V$ curves of devices containing both linkers over 15 days, introducing fresh PSI on day 8 . The lines through the data points of current density were linearly fitted in both PCBA/PSI and IQAc-PSI PV devices over 15 days as shown in Figure 7. During the first 7 days, $J_{\mathrm{SC}}$ and FF decreased gradually, at comparable rates

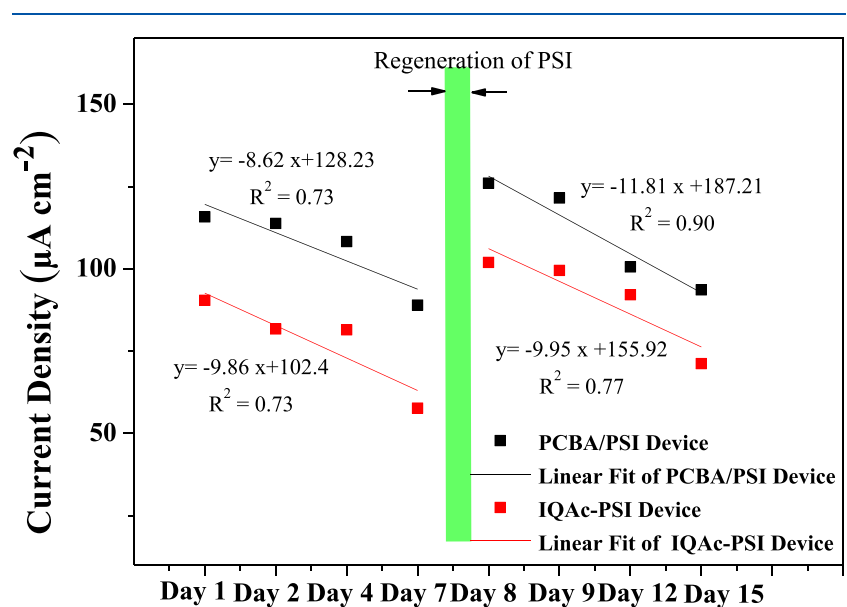

Figure 7. Comparison of photocurrent density of two sets of BPV devices fabricated using the bilayer of PCBA/PSI and the monolayer of IQAc-PSI over 15 days. On day 8, active PSI was replaced with inactive PSI. The values of photocurrent density of PCBA/PSI and IQAc-PSI BPV devices were linearly fitted as shown with black and red lines.
(Table 2), suggesting a natural deactivation/desorption process that is intrinsic to PSI and independent of the linker

Table 2. Parameters of BPV Devices over 15 days $^{a}$

\begin{tabular}{lrrr} 
& $V_{\mathrm{OC}}(\mathrm{V})$ & $J_{\text {SC }}\left(\mu \mathrm{A} / \mathrm{cm}^{2}\right)$ & FF \\
day 1 & \multicolumn{3}{c}{ PCBA/PSI } \\
day 2 & 0.58 & 115.78 & 0.294 \\
day 4 & 0.58 & 113.78 & 0.254 \\
day 7 & 0.57 & 108.27 & 0.236 \\
day 8 & 0.56 & 88.77 & 0.197 \\
day 9 & 0.58 & 126.02 & 0.300 \\
day 12 & 0.57 & 121.53 & 0.292 \\
day 15 & 0.56 & 100.60 & 0.241 \\
& 0.56 & 93.63 & 0.186 \\
day 1 & 0.50 & IQAc-PSI & \\
day 2 & 0.49 & 90.43 & 0.220 \\
day 4 & 0.48 & 81.74 & 0.208 \\
day 7 & 0.47 & 81.47 & 0.192 \\
day 8 & 0.49 & 57.66 & 0.175 \\
day 9 & 0.48 & 101.94 & 0.225 \\
day 12 & 0.47 & 99.52 & 0.218 \\
day 15 & 0.47 & 92.13 & 0.202 \\
ay & & 71.21 & 0.195
\end{tabular}

${ }^{a}$ Fresh PSI was introduced after day 7 .

used. The decreasing trend of current density can be seen in the slopes of PCBA/PSI and IQAc-PSI PV devices in Figure 7. (We observed a similar rate of decay in BPV devices comprising thiol-SAM linkers. ${ }^{15}$ ) On day 8 , we circulated a solution of active PSI through the channel containing the $\mathrm{Au}^{\mathrm{SE}}$ electrode, resulting in the recovery of device performance and increases in $J_{\mathrm{SC}}$ above their initial values, as indicated by the green line in Figure 7. In subsequent days, $J_{\mathrm{SC}}$ and FF declined as they did in the initial 7 days. This decreasing trend of current density after regeneration of fresh PSI can be seen in the slopes of both PCBA/PSI and IQAc-PSI PV devices in Figure 7. The slopes for IQAc-PSI PV devices ( -9.86 before regeneration, -9.95 after) are comparable to those of the PCBA/PSI PV devices $(-8.62$ and -11.81$)$, and there was no 
significant change in $V_{\mathrm{OC}}$ across the entire experiment. Since $V_{\mathrm{OC}}$ is almost entirely a function of the energy offsets between $\mathrm{P}_{700}^{+} / \mathrm{DCPIP}$ and $\mathrm{F}_{\mathrm{B}}$, this trend further supports the hypothesis that PSI is responsible for the photovoltaic effect and its degradation/replenishment determines the overall efficiency of the BPV devices. As the PSI denatures, $J_{S C}$ and FF decrease because there are fewer PSI complexes contributing to the photovoltaic effect, but $V_{\mathrm{OC}}$ remains constant because it is primarily a function of energy alignment and is largely unaffected by the decrease in active complexes. The photochronoamperometric measurements for devices comprising PCBA/PSI over 15 days before and after PSI regeneration under cycles of illumination $(1 \mathrm{~min})$ and dark $(4 \mathrm{~min})$ are evaluated in Figure S5.

\section{CONCLUSIONS}

Biophotovoltaic devices that use abundant, naturally occurring photosystem complexes to generate photocurrent are an intriguing approach to photovoltaic energy generation from an intrinsically low-energy manufacturing process. In nature, these protein complexes are perfectly oriented in membranes where the photochemical processes are exactly balanced by a complex arrangement of cofactors. Recreating that selfassembly-driven precision and mimicking the replacement of degraded protein are two (of many) major challenges to the design of stable, high-efficiency devices. We addressed both of these problems with a simple device design that allows the circulation of fresh PSI through devices that use linkers to direct the equilibrium of a self-assembly process toward oriented, active complexes. The performance of devices fabricated using a fullerene, PCBA, was superior to that of a peptide, IQAc, as determined via the short-circuit current, open-circuit photovoltage, and power output. The PCBA linkers self-assemble onto nanopatterned gold electrodes, simultaneously directing the orientation of PSI and facilitating the collection of photogenerated electrons. Although the absolute efficiencies remain impractically low (up to $0.0043 \%$ ), we used test designs that make no effort at light management and did not optimize the redox couple for the PCBA linker, which we intend to do in future work.

\section{ASSOCIATED CONTENT}

\section{SI Supporting Information}

The Supporting Information is available free of charge at https://pubs.acs.org/doi/10.1021/acs.langmuir.1c01542.

Atomic force microscopy (AFM) images of PCBA/PSI and IQAc-PSI, multiday current density, and output power measurements of PCBA/PSI and IQAc-PSI PV devices; comparative photochronoamperometric measurements of PCBA/PSI devices, stability of BPV devices under continuous illumination, UV-vis absorbance spectra of PCBA/PSI devices, longevity study of PCBA/PSI and IQAc-PSI BPV devices in the dark and under illumination; and control measurements (PDF)

\section{AUTHOR INFORMATION}

\section{Corresponding Author}

Ryan C. Chiechi - Stratingh Institute for Chemistry,

University of Groningen, 9747 AG Groningen, The

Netherlands; Zernike Institute for Advanced Materials, 9747

AG Groningen, The Netherlands; 10 orcid.org/0000-0002-

0895-2095; Email: r.c.chiechi@rug.nl

\section{Authors}

Nahid Torabi - Stratingh Institute for Chemistry, University of Groningen, 9747 AG Groningen, The Netherlands; Zernike Institute for Advanced Materials, 9747 AG Groningen, The Netherlands; Department of Materials Engineering, Isfahan University of Technology, Isfahan 84156-83111, Iran

Xinkai Qiu - Stratingh Institute for Chemistry, University of Groningen, 9747 AG Groningen, The Netherlands; Zernike Institute for Advanced Materials, 9747 AG Groningen, The Netherlands; Present Address: Department of Materials, University of Oxford, 16 Parks Road, Oxford OX1 3PH, United Kingdom; 이이.org/0000-0001-5857-6414

Manuel López-Ortiz - IBEC-Institut de Bioenginyeria de Catalunya, The Barcelona Institute of Science and Technology, Barcelona 08028, Spain; Network Biomedical Research Center in Biomaterials, Bioengineering and Nanomedicine (CIBER-BBN), Madrid 28029, Spain

Mark Loznik - Institute of Technical and Macromolecular Chemistry, RWTH Aachen University, 52074 Aachen, Germany; DWI-Leibniz Institute for Interactive Materials, 52056 Aachen, Germany

Andreas Herrmann - Zernike Institute for Advanced Materials, 9747 AG Groningen, The Netherlands; Institute of Technical and Macromolecular Chemistry, RWTH Aachen University, 52074 Aachen, Germany; DWI-Leibniz Institute for Interactive Materials, 52056 Aachen, Germany; (1) orcid.org/0000-0002-8886-0894

Ahmad Kermanpur - Department of Materials Engineering, Isfahan University of Technology, Isfahan 84156-83111, Iran; (C) orcid.org/0000-0001-9339-0180

Ali Ashrafi - Department of Materials Engineering, Isfahan University of Technology, Isfahan 84156-83111, Iran

Complete contact information is available at:

https://pubs.acs.org/10.1021/acs.langmuir.1c01542

\section{Notes}

The authors declare no competing financial interest.

\section{ACKNOWLEDGMENTS}

N.T. acknowledges the Zernike Institute of Advanced Materials. N.T. acknowledges financial support from the Ministry of Science, Research, and Technology of Iran (MSRT). We thank Pau Gorostiza Langa and Ricardo Zamora Brito at the Institute for Bioengineering of Catalonia (IBEC) for their cooperation during the preparation of IQAc peptides.

\section{REFERENCES}

(1) Scharber, M.; Mühlbacher, D.; Koppe, M.; Denk, P.; Waldauf, C.; Heeger, A.; Brabec, C. Design Rules for Donors in BulkHeterojunction Solar Cells-Towards 10 \% Energy-Conversion Efficiency. Adv. Mater. 2006, 18, 789-794.

(2) Clarke, T. M.; Durrant, J. R. Charge Photogeneration in Organic Solar Cells. Chem. Rev. 2010, 110, 6736-6767.

(3) Grätzel, M. Materials for Sustainable Energy; Co-Published with Macmillan Publishers Ltd.: U.K., 2010; pp 26-32.

(4) Nguyen, K.; Bruce, B. D. Growing Green Electricity: Progress and Strategies for Use of Photosystem I for Sustainable Photovoltaic Energy Conversion. Biochim. Biophys. Acta, Bioenerg. 2014, 1837, $1553-1566$.

(5) Brettel, K.; Leibl, W. Electron Transfer in Photosystem I. Biochim. Biophys. Acta, Bioenerg. 2001, 1507, 100-114.

(6) Nelson, N.; Ben-Shem, A. The Complex Architecture of Oxygenic Photosynthesis. Nat. Rev. Mol. Cell Biol. 2004, 5, 971-982. 
(7) Nelson, N.; Yocum, C. F. Structure And Function Of Photosystems I And Ii. Annu. Rev. Plant Biol. 2006, 57, 521-565.

(8) El-Mohsnawy, E.; Kopczak, M. J.; Schlodder, E.; Nowaczyk, M.; Meyer, H. E.; Warscheid, B.; Karapetyan, N. V.; Rögner, M. Structure and Function of Intact Photosystem 1 Monomers From the Cyanobacterium Thermosynechococcus elongatus. Biochemistry 2010, 49, 4740-4751.

(9) Gordiichuk, P. I.; Wetzelaer, G.-J. A. H.; Rimmerman, D.; Gruszka, A.; de Vries, J. W.; Saller, M.; Gautier, D. A.; Catarci, S.; Pesce, D.; Richter, S.; Blom, P. W. M.; Herrmann, A. Solid-State Biophotovoltaic Cells Containing Photosystem I. Adv. Mater. 2014, 26, 4863-4869.

(10) Gizzie, E. A.; Niezgoda, J. S.; Robinson, M. T.; Harris, A. G.; Jennings, G. K.; Rosenthal, S. J.; Cliffel, D. E. Photosystem IPolyaniline/TiO2 Solid-State Solar Cells: Simple Devices for Biohybrid Solar Energy Conversion. Energy Environ. Sci. 2015, 8, 3572-3576.

(11) Beam, J. C.; LeBlanc, G.; Gizzie, E. A.; Ivanov, B. L.; Needell, D. R.; Shearer, M. J.; Jennings, G. K.; Lukehart, C. M.; Cliffel, D. E. Construction of a Semiconductor-Biological Interface for Solar Energy Conversion: P-Doped Silicon/Photosystem I/Zinc Oxide. Langmuir 2015, 31, 10002-10007.

(12) Das, R.; Kiley, P. J.; Segal, M.; Norville, J.; Yu, A. A.; Wang, L.; Trammell, S. A.; Reddick, L. E.; Kumar, R.; Stellacci, F.; Lebedev, N.; Schnur, J.; Bruce, B. D.; Zhang, S.; Baldo, M. Integration of Photosynthetic Protein Molecular Complexes in Solid-State Electronic Devices. Nano Lett. 2004, 4, 1079-1083.

(13) Ciornii, D.; Riedel, M.; Stieger, K. R.; Feifel, S. C.; Hejazi, M.; Lokstein, H.; Zouni, A.; Lisdat, F. Bioelectronic Circuit on a 3D Electrode Architecture: Enzymatic Catalysis Interconnected With Photosystem I. J. Am. Chem. Soc. 2017, 139, 16478-16481.

(14) Passantino, J. M.; Wolfe, K. D.; Simon, K. T.; Cliffel, D. E.; Jennings, G. K. Photosystem I Enhances the Efficiency of a Natural, Gel-Based Dye-Sensitized Solar Cell. ACS Appl. Bio Mater. 2020, 3, $4465-4473$.

(15) Qiu, X.; Ocampo, O. C.; de Vries, H. W.; van Putten, M.; Loznik, M.; Herrmann, A.; Chiechi, R. C. Self-Regenerating Soft Biophotovoltaic Devices. ACS Appl. Mater. Interfaces 2018, 10, 37625-37633.

(16) Wolfe, K. D.; Dervishogullari, D.; Stachurski, C. D.; Passantino, J. M.; Jennings, G. K.; Cliffel, D. E. Photosystem I Multilayers Within Porous Indium Tin Oxide Cathodes Enhance Mediated Electron Transfer. ChemElectroChem 2020, 7, 596-603.

(17) Ciesielski, P. N.; Hijazi, F. M.; Scott, A. M.; Faulkner, C. J.; Beard, L.; Emmett, K.; Rosenthal, S. J.; Cliffel, D.; Jennings, G. K. Photosystem I-Based biohybrid photoelectrochemical cells. Bioresour. Technol. 2010, 101, 3047-3053.

(18) Tschörtner, J.; Lai, B.; Krömer, J. O. Biophotovoltaics: Green Power Generation From Sunlight and Water. Front. Microbiol. 2019, 10, No. 866

(19) Sokol, K. P.; Mersch, D.; Hartmann, V.; Zhang, J. Z.; Nowaczyk, M. M.; Rögner, M.; Ruff, A.; Schuhmann, W.; Plumeré, N.; Reisner, E. Rational Wiring of Photosystem II to Hierarchical Indium Tin Oxide Electrodes Using Redox Polymers. Energy Environ. Sci. 2016, 9, 3698-3709.

(20) Takekuma, Y.; Ikeda, N.; Kawakami, K.; Kamiya, N.; Nango, M.; Nagata, M. Photocurrent Generation by a Photosystem I-NiO Photocathode for a P-Type Biophotovoltaic Tandem Cell. RSC Adv. 2020, 10, 15734-15739.

(21) Takeuchi, R.; Suzuki, A.; Sakai, K.; Kitazumi, Y.; Shirai, O.; Kano, K. Construction of Photo-Driven Bioanodes Using Thylakoid Membranes and Multi-Walled Carbon Nanotubes. Bioelectrochemistry 2018, 122, 158-163.

(22) Pankratov, D.; Zhao, J.; Nur, M. A.; Shen, F.; Leech, D.; Chi, Q.; Pankratova, G.; Gorton, L. The Influence of Surface Composition of Carbon Nanotubes on the Photobioelectrochemical Activity of Thylakoid Bioanodes Mediated by Osmium-Complex Modified Redox Polymer. Electrochim. Acta 2019, 310, 20-25.
(23) Feifel, S. C.; Stieger, K. R.; Hejazi, M.; Wang, X.; Ilbert, M.; Zouni, A.; Lojou, E.; Lisdat, F. Dihemic C4-Type Cytochrome Acting as a Surrogate Electron Conduit: Artificially Interconnecting a Photosystem I Supercomplex With Electrodes. Electrochem. Commun. 2018, 91, 49-53.

(24) Stieger, K. R.; Feifel, S. C.; Lokstein, H.; Hejazi, M.; Zouni, A.; Lisdat, F. Biohybrid Architectures for Efficient Light-to-Current Conversion Based on Photosystem I Within Scalable 3D Mesoporous Electrodes. J. Mater. Chem. A 2016, 4, 17009-17017.

(25) Ciornii, D.; Kölsch, A.; Zouni, A.; Lisdat, F. A PrecursorApproach in Constructing 3D ITO Electrodes for the Improved Performance of Photosystem I-Cyt C Photobioelectrodes. Nanoscale 2019, 11, 15862-15870.

(26) Stieger, K. R.; Feifel, S. C.; Lokstein, H.; Lisdat, F. Advanced Unidirectional Photocurrent Generation via Cytochrome $\mathrm{C}$ as Reaction Partner for Directed Assembly of Photosystem I. Phys. Chem. Chem. Phys. 2014, 16, 15667-15674.

(27) Szalkowski, M.; Olmos, J. D. J.; Buczyńska, D.; Maćkowski, S.; Kowalska, D.; Kargul, J. Plasmon-Induced Absorption of Blind Chlorophylls in Photosynthetic Proteins Assembled on Silver Nanowires. Nanoscale 2017, 9, 10475-10486.

(28) Gordiichuk, P.; Pesce, D.; Ocampo, O. E. C.; Marcozzi, A.; Wetzelaer, G.-J. A. H.; Paul, A.; Loznik, M.; Gloukhikh, E.; Richter, S.; Chiechi, R. C.; Herrmann, A. Orientation and Incorporation of Photosystem I in Bioelectronics Devices Enabled by Phage Display. Adv. Sci. 2017, 4, No. 1600393.

(29) Castañeda Ocampo, O. E.; Gordiichuk, P.; Catarci, S.; Gautier, D. A.; Herrmann, A.; Chiechi, R. C. Mechanism of OrientationDependent Asymmetric Charge Transport in Tunneling Junctions Comprising Photosystem I. J. Am. Chem. Soc. 2015, 137, 8419-8427.

(30) Gunther, D.; LeBlanc, G.; Prasai, D.; Zhang, J. R.; Cliffel, D. E.; Bolotin, K. I.; Jennings, G. K. Photosystem I on Graphene as a Highly Transparent, Photoactive Electrode. Langmuir 2013, 29, 4177-4180.

(31) Manocchi, A. K.; Baker, D. R.; Pendley, S. S.; Nguyen, K.; Hurley, M. M.; Bruce, B. D.; Sumner, J. J.; Lundgren, C. A. Photocurrent Generation From Surface Assembled Photosystem I on Alkanethiol Modified Electrodes. Langmuir 2013, 29, 2412-2419.

(32) Badura, A.; Kothe, T.; Schuhmann, W.; Rögner, M. Wiring Photosynthetic Enzymes to Electrodes. Energy Environ. Sci. 2011, 4, 3263.

(33) Pachoumi, O.; Bakulin, A. A.; Sadhanala, A.; Sirringhaus, H.; Friend, R. H.; Vaynzof, Y. Improved Performance of $\mathrm{ZnO} /$ Polymer Hybrid Photovoltaic Devices by Combining Metal Oxide Doping and Interfacial Modification. J. Phys. Chem. C 2014, 118, 18945-18950.

(34) Lee, E. J.; Heo, S. W.; Han, Y. W.; Moon, D. K. An Organicinorganic Hybrid Interlayer for Improved Electron Extraction in Inverted Polymer Solar Cells. J. Mater. Chem. C 2016, 4, 2463-2469.

(35) Thu, C.; et al. Role of the Metal-Oxide Work Function on Photocurrent Generation in Hybrid Solar Cells. Sci. Rep. 2018, 8, No. 3559.

(36) Aryal, U. K.; Arivunithi, V. M.; Reddy, S. S.; Kim, J.; Gal, Y.-S.; Jin, S.-H. Efficient Dual Cathode Interfacial Layer for High Performance Organic and Perovskite Solar Cells. Org. Electron. 2018, 63, 222-230.

(37) Alshanableh, A.; Tan, S. T.; Yap, C. C.; Lee, H. B.; Oleiwi, H. F.; Hong, K. J.; Jumali, M. H. H.; Yahaya, M. Surface Engineering of $\mathrm{ZnO}$ Nanorod for Inverted Organic Solar Cell. Mater. Sci. Eng., B 2018, 238-239, 136-141

(38) Hummelen, J. C.; Knight, B. W.; LePeq, F.; Wudl, F.; Yao, J.; Wilkins, C. L. Preparation and Characterization of Fulleroid and Methanofullerene Derivatives. J. Org. Chem. 1995, 60, 532-538.

(39) Ortiz, M. L.; Zamora, R. A.; Giannotti, M. I.; Hu, C.; Croce, R.; Gorostiza, P. Distance and Potential Dependence of Charge Transport Through the P700 Reaction Center of Photosystem I. ChemRxiv 2021, DOI: 10.26434 / chemrxiv. 14556048.

(40) Qiu, X.; Ivasyshyn, V.; Qiu, L.; Enache, M.; Dong, J.; Rousseva, S.; Portale, G.; Stöhr, M.; Hummelen, J. C.; Chiechi, R. C. Thiol-Free Self-Assembled Oligoethylene Glycols Enable Robust Air-Stable Molecular Electronics. Nat. Mater. 2020, 19, 330-337. 
(41) Vaynzof, Y.; Kabra, D.; Zhao, L.; Ho, P. K. H.; Wee, A. T.-S.; Friend, R. H. Improved Photoinduced Charge Carriers Separation in Organic-Inorganic Hybrid Photovoltaic Devices. Appl. Phys. Lett. 2010, 97, No. 033309.

(42) Vaynzof, Y.; Bakulin, A. A.; Gélinas, S.; Friend, R. H. Direct Observation of Photoinduced Bound Charge-Pair States at an Organic-Inorganic Semiconductor Interface. Phys. Rev. Lett. 2012, 108, No. 246605.

(43) Lee, I.; Lee, J. W.; Greenbaum, E. Biomolecular Electronics: Vectorial Arrays of Photosynthetic Reaction Centers. Phys. Rev. Lett. 1997, 79, 3294-3297.

(44) Faulkner, C. J.; Lees, S.; Ciesielski, P. N.; Cliffel, D. E.; Jennings, G. K. Rapid Assembly of Photosystem I Monolayers on Gold Electrodes. Langmuir 2008, 24, 8409-8412.

(45) Rippka, R.; Stanier, R. Y.; Deruelles, J.; Herdman, M.; Waterbury, J. B. Generic Assignments, Strain Histories and Properties of Pure Cultures of Cyanobacteria. Microbiology 1979, 111, 1-61.

(46) Mukherjee, D.; May, M.; Vaughn, M.; Bruce, B. D.; Khomami, B. Controlling the Morphology of Photosystem I Assembly on ThiolActivated Au Substrates. Langmuir 2010, 26, 16048-16054.

(47) So, J.; Dickey, M. Inherently Aligned Microfluidic Electrodes Composed of Liquid Metal. Lab Chip 2011, 11, 905-911.

(48) Dickey, M.; Weiss, E.; Smythe, E.; Chiechi, R.; Capasso, F.; Whitesides, G. Fabrication of Arrays of Metal and Metal Oxide Nanotubes by Shadow Evaporation. ACS Nano 2008, 2, 800-808.

(49) Petrova, A.; Mamedov, M.; Ivanov, B.; Semenov, A.; Kozuleva, M. Effect of Artificial Redox Mediators on the Photoinduced Oxygen Reduction by Photosystem I Complexes. Photosynth. Res. 2018, 137, 421-429.

(50) Santiago, A. R. P.; Fernandez-Delgado, O.; Gomez, A.; Ahsan, M. A.; Echegoyen, L. Fullerenes as Key Components for LowDimensional (Photo)electrocatalytic Nanohybrid Materials. Angew. Chem., Int. Ed. 2021, 133, 124-143.

(51) Pan, Y.; Liu, X.; Zhang, W.; Liu, Z.; Zeng, G.; Shao, B.; Liang, Q.; He, Q.; Yuan, X.; Huang, D.; Chen, M. Advances in photocatalysis based on fullerene $\mathrm{C} 60$ and its derivatives: Properties, mechanism, synthesis, and applications. Appl. Catal., B 2020, 265, No. 118579.

(52) Blom, P.; Mihailetchi, V.; Koster, L.; Markov, D. Device Physics of Polymer:Fullerene Bulk Heterojunction Solar Cells. Adv. Mater. 2007, 19, 1551-1566.

(53) Li, S.; Lei, M.; Lv, M.; Watkins, S. E.; Tan, Z.; Zhu, J.; Hou, J.; Chen, X.; Li, Y. [6, 6]-Phenyl-C61-Butyric Acid Dimethylamino Ester as a Cathode Buffer Layer for High-Performance Polymer Solar Cells. $A d v$. Energy Mater. 2013, 3, 1569-1574.

(54) Tel-Vered, R.; Willner, I. Photo-Bioelectrochemical Cells for Energy Conversion, Sensing, and Optoelectronic Applications. ChemElectroChem 2014, 1, 1778-1797.

(55) Tsarev, S.; Dubinina, T. S.; Luchkin, S. Y.; Zhidkov, I. S.; Kurmaev, E. Z.; Stevenson, K. J.; Troshin, P. A. Phenyl-C61-butyric Acid as an Interface Passivation Layer for Highly Efficient and Stable Perovskite Solar Cells. J. Phys. Chem. C 2020, 124, 1872-1877.

(56) Mishra, A.; Bäuerle, P. Small Molecule Organic Semiconductors on the Move: Promises for Future Solar Energy Technology. Angew. Chem., Int. Ed. 2012, 51, 2020-2067.

(57) Nelson, J. Polymer:fullerene bulk heterojunction solar cells. Mater. Today 2011, 14, 462-470.

(58) Nakanishi, W.; Minami, K.; Shrestha, L. K.; Ji, Q.; Hill, J. P.; Ariga, K. Bioactive nanocarbon assemblies: Nanoarchitectonics and applications. Nano Today 2014, 9, 378-394.

(59) Yan, J.; Saunders, B. R. Third-generation solar cells: a review and comparison of polymer: fullerene, hybrid polymer and perovskite solar cells. RSC Adv. 2014, 4, 43286-43314.

(60) Shi, X.-Q.; Hove, M. A. V.; Zhang, R.-Q. Survey of Structural and Electronic Properties of C60 on Close-Packed Metal Surfaces. J. Mater. Sci. 2012, 47, 7341-7355.

(61) Cho, N.-K.; Na, H.-J.; Yoo, J.; Kim, Y. S. Long-term stability in $\gamma$-CsPbI3 perovskite via an ultraviolet-curable polymer network. Commun. Mater. 2021, 2, No. 30.
(62) Suresh, L.; Vaghasiya, J. V.; Nandakumar, D. K.; Wu, T.; Jones, M. R.; Tan, S. C. High-Performance UV Enhancer Molecules Coupled with Photosynthetic Proteins for Ultra-Low-Intensity UV Detection. Chem 2019, 5, 1847-1860.

(63) Long, S. P.; Humphries, S.; Falkowski, P. G. Photoinhibition of Photosynthesis in Nature. Annu. Rev. Plant Physiol. Plant Mol. Biol. 1994, 45, 633-662.

(64) Allahverdiyeva, Y.; Aro, E.-M. Photosynthesis; Springer: Netherlands, 2011; pp 275-297.

(65) Paul, N.; Suresh, L.; Vaghasiya, J. V.; Yang, L.; Zhang, Y.; Nandakumar, D. K.; Jones, M. R.; Tan, S. C. Self-powered all weather sensory systems powered by Rhodobacter sphaeroides protein solar cells. Biosens. Bioelectron. 2020, 165, No. 112423.

(66) Liu, J.; Friebe, V. M.; Frese, R. N.; Jones, M. R. Polychromatic solar energy conversion in pigment-protein chimeras that unite the two kingdoms of (bacterio)chlorophyll-based photosynthesis. Nat. Commun. 2020, 11, No. 1542.

(67) Molamohammadi, S.; Jalili, Y. S.; Riazi, G. Photosystem I application in biohybrid polymer solar cells. AIP Adv. 2018, 8, No. 095319. 